\title{
Sport Pour Tous \& Socialisation en Tunisie
}

\author{
Abdelkerim Boubaker \\ Enseignant chercheur, membre de l'unité de recherche en psychologie du sport à l'institut supérieur du sport et \\ de l'éducation physique. Ksar Said Tunis
}

\begin{abstract}
RESUME : La sociabilité développée au sein des clubs sport pour tous( SPT) serait commandée à l'origine par une finalité provoquée par les exigences de la vie; on peut déduire que la sociabilité du SPT sans cesse activée par ce système d'échange généralisé double d'un temps de pratique collective, sportive et d'ambiance de gaieté, se pérennise également grâce à une mémoire partagée; d'où on peut dire que la pratique des activités physiques libres a une influence positive sur la socialisation puisqu'elle favorise la communication inter-pratiquant et met en valeurs les relations humaines a travers l'instauration d'une bonne ambiance de pratique par le biais des compétitions organisés ainsi que l'acquisition de différentes valeurs morales comme la solidarité, la responsabilité l'autonomie et l'entre-aide ; et n'oublions pas le rôle que joue les programmes SPT dans l'acquisition des habiletés mentales et physiques chez l'individu.

Aussi, l'intégration des pratiquants de SPT dans la société par la participation active au monde du travail et la persistance des liens de solidarité entretenus entre les individus en dehors de la sphère sportive, crée des réseaux d'échanges, d'informations, de biens et de services, en même temps que des obligations et une interdépendance entre les bénéficiaires.

La connaissance de ces réseaux et de leur fonctionnement permet de comprendre le système de relations existant dans le SPT, et qui donnent à ce fameux esprit SPT, sa signification sociale, par là on peut dire que la pratique du SPT a un impact meilleur que la pratique du sport civil puisqu'elle favorise l'intégration dans le groupe car c'est un espace où se développent les logiques participatives ainsi qu'elle favorise la consolidation des relations sociales, n'oublions pas qu'elle aide dans la gestion et la réduction du stress, de l'anxiété, de la dépression et accroit la capacité de production de l'individu dans son travail.
\end{abstract}

Mots clés : sport pour tous, la pratique sportive, socialisation, activités physiques et sportives, socialisation sportive

\section{Introduction}

La socialisation c'est : " transformer un être asociale en un être sociale qui a intériorisé un certains nombre de règles, de normes et de valeurs ce qui forme alors une certaine cohésion sociale $»^{1}$, on peut donc résumer cela en disant que la socialisation c'est la capacité pour un individu à vivre avec d'autre individu dans une même société. L'individu va subir tout au long de sa vie des apprentissages qui vont le mener à cette socialisation. La socialisation remplirait également deux fonctions qui sont de favoriser l'adaptation de chaque individu à la vie sociale dans un premier temps et dans un second temps de favoriser et maintenir un degré de cohésion entre les membres de la société. La socialisation d'un individu ne se fait pas toute seule et pour ce faire l'individu doit traverser différents milieux, différentes instances. Il y tout d'abord la famille, considéré comme la première instance de socialisation, la famille est la plus importante instance de socialisation dans la vie d'un individu. Puis vient l'école en second lieu qui tiens de plus en plus une place déterminante dans la socialisation de l'enfant car celui-ci tend à y entrer de plus en plus tôt et à la quitter de plus en plus tard. Ensuite c'est le travail qui prime comme troisième instance de socialisation. En quatrième nous avons les groupes de pairs (entourage, famille, etc.) qui entre dans le processus de socialisation de l'individu et en dernière instance on y trouve les médias qui tendent à avoir de plus en plus d'impact sur la socialisation des jeunes de nos jours, notamment par la révolution du système informatique.

Le sport prend une place importante (éducative) dans la socialisation du jeune car c'est une discipline obligatoire. On pourrait définir le sport en sociologie comme étant une « sous-instance » de socialisation que l'on tend à retrouver un peu dans toutes les instances de socialisations vu précédemment. Le sport est aussi défini comme étant un «agent» de socialisation. Plusieurs définitions du sport existent. De fait, nous retiendrons que le sport est une activité physique, codifiée, institutionnelle et compétitive. Par extension on

\footnotetext{
1 - R.Boudon (1997), dictionnaire de sociologie, http://aees.free.fr/docs\%20liens/cours/12\%20semestre\%202/SHS\%20
} 
appelle sport toute activité relevant d'une fédération sportive, quelque soit la façon de la pratiquer ${ }^{2}$ selon PARLEBAS P. (1981).

\section{Problématique}

Il serait donc intéressant de se demander si le sport ne serait pas un peu trop survalorisé en terme de socialisation car, outre son côté socialisant, il crée tout de même des déviances comme le hooliganisme, le dopage, la violence qui est devenue une réponse aux valeurs du sport...

La mauvaise pratique du sport peut causer de la déception Les valeurs démontrées dans certains sports professionnels ne sont pas les exemples à suivre, l'actualité en témoigne chaque jour. La Tunisie compte aujourd'hui plusieurs clubs et associations sportives. Face à l'accroissement de leurs dépenses de fonctionnement et du nombre de jeunes pratiquant le sport, les structures sportives font de plus en plus appel aux bénévoles (denrées qui se raréfient), pour des tâches d'encadrement, de logistique et d'administration, avec un maître mot pour tous, la passion...

Alors, y-a-t-il une solution meilleure à fin de sauver les valeurs du sport ?

\section{HYPOTHESES}

H.1 : nous admettons que la pratique des activités physiques et sportives libres a une influence positive sur la socialisation.

H.2 : la pratique du sport pour tous a un impact meilleur sur la socialisation que la pratique du sport civil.

\section{BUTS DE LA RECHERCHE}

Nous avons adopté cette recherche pour vérifier si la socialisation de la pratique sportive libre a un impact meilleur concernant la protection des valeurs du sport que le sport civil.

\section{Le Sport pour Tous}

\section{LES CONCEPTS}

«Le Sport pour Tous, pour le Conseil de l'Europe ${ }^{3}$, c'est de permettre à tous, personnes des deux sexes et de tous les groupes d'âges, de conserver les capacités physiques et psychiques nécessaires à la survie et de préserver l'espèce humaine de toute dégradation $»$.

« Le mot sport est à comprendre dans le sens moderne, c'est-à-dire, celui de l'activité physique libre, spontanée, pratiquée pendant les loisirs et englobant les sports proprement dit et des activités diverses, pourvues qu'elles exigent un certain effort $»^{4}$

Affirmations révélées par le Cleaning House ${ }^{5}$ afin de mieux situer le SPT :

$>$ «L'activité physique n'est pas un acte gratuit, c'est un acte de vie».

$>$ «Le Sport pour Tous prouve que le sport n'appartiens pas à une élite ou à une masse mais à tous ».

$>$ «Le Sport pour Tous a une vocation qui dépasse largement le domaine purement physique. Il peut apporter une contribution essentielle au développement et à l'expression de la personnalité et à la définition de l'humain dans une civilisation mécanisée ».

$>$ «Le Sport pour tous n'est pas la panacée mais il est un des moyens sûrs et peu coûteux pour vivre heureux et longtemps».

M.P.BERNARD a mis l'accent dans un remarquable article ${ }^{6}$ sur l'ambigüité de la définition du SPT en insistant sur la nécessité d'établir une certaine continuité entre les différentes formes de pratiques, continuité évolutive qui aboutirait jusqu'au l'accès aux performances.

Donc, le SPT n'est pas seulement l'activité physique dans sa forme ludique, gratuite, librement consentie, mais aussi le sport traditionnel dans sa forme agonale, compétitive, ouverte à tous selon de nouvelles formules récréatives qu'il faut imaginer, d'activités nécessaires, susceptibles de satisfaire les aspirations des pratiquants.

Le SPT est une pratique des jeunes gens d'âge un peu avancé (22-70 ans) dans les villes ou les campagnes et de toutes les couches de la société. C'est une pratique d'errance, d'incessants déplacements dans l'espace public qui obligent l'observateur lui-même à se transformer en errant, en aventurier. La sociologie du SPT est une sociologie qui utilise des méthodes " ethnologiques », c'est-à-dire qui privilégie le regard et l'écoute ; une sociologie du quotidien qui s'écrit avec des mots et des images, qui entendent restituer le sens que les acteurs attribuent à leurs actes, sans oublier les enjeux sociaux qui se dressent derrière les pratiques du SPT.

\footnotetext{
2 - Parlebas, Contribution à un lexique commenté en science de l'action motrice, Paris, INSEP, 1981.

3 - Document du Conseil de l'Europe, Charte Européenne du Sport pour Tous, p. 3 du document.

${ }^{4}$ - Charte Européenne du Sport pour Tous, document CCC/EES (68) 10 rév. II Strasboug 1979.

5 - Cleaning House:Centre d'information sportive du Conseil de l'Europe, fiches d'informations 1974, fiche n 3 .

${ }^{6}$ - Michel Paul BERNARD : professeur Bulletin FIEP, Mars 1981, pages 45 et suivantes.
} 
Différenciés ou mêlés, jeu, sport, loisirs, mode de vie, le SPT se retrouve dans tous ces mots, mais il en bouscule souvent les sens conventionnels; il fait émerger d'autres sens qui, à leur tour, bousculent les idées reçues.

C'est un espace de convivialité, de l'entre-soi, usant d'une forme de sociabilité pour les pratiquants. Cette sociabilité qui peut être organisée ou spontanée, présente un caractère différent selon les lieux, selon les instants. Il met davantage en avant les valeurs d'épanouissement, d'amusement, de coopération, d'entraide et de solidarité. La sociabilité développée au sein du SPT se veut démocratique, sans parvenir à l'être toujours. Simmel précise à ce propos que « les aptitudes exceptionnelles et les mérites de l'individu n'ont aucun rôle à jouer dans la sociabilité. »?

La diversité des définitions du sport s'est accentuée par l'introduction d'un nouveau concept social, culturel et humaniste du sport c'est le Sport pour Tous, natif de la civilisation industrielle et du développement technologique. Mais, ce concept répondra-t-il aux différentes formes du sport que ce soit agonale, compétitive, traditionnelle ou bien ludique, récréative de loisirs, de détente et du jeu ?

La mise en œuvre de cette conception révèle d'un besoin, de la nécessité de rétablir l'équilibre d'une société menacée dans sa cohésion sociale par les avatars de la modernité tels que l'isolement, la marginalité, le déséquilibre, la délinquance et la criminalité ; et donc c'est une manière de compenser les dangers biologiques d'une sédentarité due au manque de mouvement qui réduit la circulation périphérique du sang et augmente la fatigue musculaire et les perturbations du métabolisme et des affectations cardiaques.

Comme le souligne A. CHAHED « le Sport pour Tous se propose de libérer ce corps, de le prémunir, de faire reculer tous les signes de fatigue et de nous le faire découvrir comme le pivot de toute notre vie ${ }^{8}$ donc, c'est une solution adéquate et efficace de réduire et anéantir les effets néfastes des conditions de vie verticale dans les immeubles et les zones urbaines (exode rural ou changement de résidence) d'où le rejet, l'isolement et la solitude cèdent le pas aux relations humaines à la chaleur de vie en groupe et surtout aux sentiments de bien être.

Le SPT ${ }^{9}$ c'est un signe de développement et d'essoufflement du sport compétitif qui ne répond plus aux besoins de la société moderne; il exprime non seulement son inquiétude et un désarroi devant les agressions de la civilisation technologique, mais aussi le besoin de défendre de l'être humain et la recherche d'une vie meilleure. Activités physiques et sportives (APS)

Activités physiques et sportives (APS) : terme générique amalgamant l'ensemble des situations motrices dans lesquelles un sujet cherche à réaliser une/des habilité(s) requérant l'expression de processus mécaniques, énergétiques et coordonnatifs. Cependant, la variété et la diversité des pratiques physiques nécessitent de distinguer deux catégories

1) L'activité physique : elle est "l'interaction entre l'individu et sa motricité" (Paddick). Elle peut être exercée à titre utilitaire et professionnel (ex: manutentionnaire, déménageur...) mais également dans le cadre privé des loisirs (ex: baignade, sortie en vélo...), les motivations sous-tendues pouvant être: le besoin d'affiliation, la forme physique, la recherche du vertige, la conquête d'une esthétique, la recherche d'une ascèse dans l'effort, un éventuel effet cathartique (défoulement)... En ce sens, le jogging matinal dominical, la sortie cycliste entre amis ou la baignade épisodique sont des activités physiques. Pour quelles deviennent Activité Sportives, elles doivent intégrer d'autres dimensions et notamment la compétition.

2) L'activité sportive : (le sport), "ensemble des situations motrices d'affrontement codifié dont les formes compétitives sont institutionnalisée" (Parlebas). Le sport est la version évoluée de l'agôn de Caillois, faisant apparaitre trois dimensions fondamentales: a/ une situation motrice (ainsi ni les cartes ni les échecs ne sont des sport) $\mathrm{b} /$ un affrontement codifié (adaptation de règles uniformisées). c/ une compétition institutionnalisée (organisée par les fédérations) Ainsi le sport intègre l'affrontement compétitif comme élément centrale et essentiel, instituant des valeurs, des normes, des qualifications et des connaissances spécifiques. ${ }^{10}$

\section{Socialisation}

C'est le processus par lequel l'individu apprend et intériorise différents éléments de culture de son groupe, ce qui lui permet de former sa propre personnalité sociale et de s'adapter au groupe dans lequel il vit.

On est socialisé par rapport au groupe auquel on appartient. Mais un individu peut appartenir à plusieurs groupes.Exemple : l'enfant est socialisé par la famille, l'école, les amis, le club de sport.

\footnotetext{
7 - Simmel.G, sociologie et épistémologie, PUF, Paris, 1981 p 126.

8 - Abdelwahab CHAHED, Pour une politique Tunisienne de Sport pour Tous, Tunis-La Kasbah 1986, p. 13.

9 - Sport pour Tous

10 - Définition intégrale, tiré du dictionnaire des APS, éditions Amphora 1998.
} 
Par la socialisation l'individu apprend à vivre en société. Ainsi sans cette socialisation il est difficile de vivre en société.

Définition de Guy Rocher : «Ensemble de processus par lequel l'individu apprend des manières de faire, d'agir, de penser, de sentir c'est à dire la culture ${ }^{11}$.

Dans le premier paragraphe les termes les plus importants sont «tout au long de la vie », « culture » et « personnalité sociale »= à la personnalité psychique s'ajoutent des éléments de la sociabilité jusqu'à ce qu'ils deviennent partie intégrante de l'individu.

La socialisation n'uniformise pas totalement. Tout au long de sa vie, l'homme évolue, sa personnalité sociale aussi évolue : l'homme est unique et uniforme. Le résultat est complexe.

\section{Socialisation Sportive}

La socialisation est « Le processus par lequel les individus apprennent les modes d'agir et de penser leur environnement, les intériorisent en les intégrant à leur personnalité et deviennent membres de groupes où ils acquièrent un statut spécifique »; ainsi, « Le club sportif est un espace de sociabilité. Il renforce les liens avec le groupe social, crée des réseaux de solidarité locale et de sentiments d'appartenance ${ }^{12}$.

« Le sport peut être, pour les jeunes en difficulté, un lieu de resocialisation par dépassement des différences, retournement des stigmates, réappropriation d'identités positives $»^{13}$.

« Nous définissons provisoirement la sociabilité comme les formes d'expressions et la régularité des manifestations et des relations qu'un individu entretient avec autrui dans un domaine constitué objectivable. $»^{14}$. Le sport prend de plus en plus de place dans notre société. L'ambiance de nos villes peut être complètement transformée les soirs de grands matchs, et les Jeux olympiques sont un événement digne de la mondialisation. Les médias l'ont bien compris : il y a des journaux consacrés aux sports, des chaines télévisées thématiques.

\section{- LE SPORT POUR TOUS EN TUNISIE}

Le sport en Tunisie, qui émergea réellement durant le protectorat français, est marqué par la domination du football, tant en termes de couverture médiatique qu'en termes de succès populaire.

Toutefois, des sports comme le volley-ball (huit victoires de l'équipe nationale en championnat d'Afrique) ou le handball (sept victoires de l'équipe nationale en championnat d'Afrique) figurent également parmi les sports les plus représentés même si des sports moins connus sont plus pratiqués par les Tunisiens, notamment les arts martiaux (taekwondo, judo et karaté), l'athlétisme, la natation voire le tennis. D'autres grands sports comme le cyclisme, l'aviron ou la voile sont en revanche moins représentés, faute d'infrastructures, d'équipement et d'intérêt médiatique suffisant.

\section{SPT : NAISSANCE EN TUNISIE ${ }^{15}$ :}

Les activités Sport et Travail furent développées depuis novembre 1971 qui semble être la date de la création de la Fédération Tunisienne Sport et Travail actuellement (FTST), l'Association Nationale Sport Culture et Travail (ANSCT). Celle-ci au contact d'organismes similaires étrangers comme la Fédération Sportive et gymnique du Travail de France, l'Union Italienne du Sport Populaire, la Fédération Française de l'Entrainement Physique dans le Monde Moderne et la Centrale des Fédérations Sportives Belges se pencha avec beaucoup d'intérêt sur les expériences en matière de Sport pour Tous.

Elle fut acquise et très vite entrer dans les mœurs sportives tunisiennes le loisir sportif. A partir de 1974 le concept SPT était fréquemment utilisé dans les jargons de ses responsables pour devenir un de leurs objectifs à long terme, une activité attirante, fascinante et exaltante. Ils ont voulu forcer leurs destin sans le préparer longtemps à l'avance, sans appui logistique ni soutiens malgré leurs faibles moyens et leur manque d'expérience.

Un séminaire organisé du 25 au 27 Juin 1976 à Bir El Bey devait encore confirmer l'orientation prise par $1^{\prime}$ ANSCT $^{16}$ depuis 1975. Les résolutions de la commission SPT ont adopté une définition, proposé le lancement

11 - GUY ROCHER, Introduction à la sociologie générale, Montréal (HMH) ,1968.

12 - Fize, Michel (1993). Socialisation- sociabilité : qu'en dire ?, dans "Du stade au quartier : Le rôle du sport dans l'intégration sociale des jeunes ", p.39.

13 - Dubar, Claude (1993). L'autre jeunesse : d'autres voies de socialisation? Dans "Du stade au quartier : Le rôle du sport dans l'intégration sociale des jeunes ", p.36.

14 - Acte du colloque des 14 et 15 octobre 1993 à Bordeaux. (1995) Sport, relations sociales et action collective. Chapitre sur " L'Espace des sociabilités féminines en sports collectifs ", par Christine Mennesson. Talence : éditions de la maison des sciences de l'homme d'aquitaine. P. 231-237.

15 -Abdelwahab CHAHED,Pour une politique Tunisienne de Sport pour Tous, Tunis-La Kasbah 1986, p33 à 38.

16 - Association Nationale Sport, Culture et Travail. 
d'une compagne dénommée Slim ${ }^{17}$ qui rimait bien avec Trim ; ils ont par ailleurs proposé la création d'un club Slim qui aurait pour objet de proposer des activités physiques et sportives simples, variées, non compétitives et sans classement non seulement aux travailleurs, mais aussi à leurs familles. Ils ont même énuméré certaines centres d'entres elles et tracé les grandes lignes de la compagne de sensibilisation.

De 1976 à 1984, les dirigeants de l'ANSCT organisèrent des journées de SPT notamment à partir de 1981, régionalisées, surtout d'athlétisme et de cyclisme, drainant la grande foule, créant une ambiance de fête. Ce fût un moyen efficace de sensibilisation, une manière de brancher les autorités, d'intéresser la T.V. qui en diffusera des séquences et de mettre en lice le département de la tutelle.

Par ailleurs, la Revue Sport, Culture et Travail organe officielle de l'ANSCT a continué (1979-1980) à accorder au SPT une place de choix dans ses colonnes et en propager les principes.

C'est seulement en 1981 que la Direction de l'Education Physique et des Sports du Ministère de la Jeunesse et des Sports s'est mise au diapason et a préparé un rapport complet sur le SPT qui a été proposé à la Conférence Nationale sur le Développement du Sport (Août 1981) ${ }^{18}$ qui l'a pris en charge en tant que résolution. La distinction entre Sport d'Elite, Sport de Masse et SPT fut établie. Un Conseil National Sport pour Tous n'a pas retenu le principe de la création d'un service « SPT » au sein de la Direction de l'EPS ${ }^{19}$.

L'article 24 du décret 83/1189 du 14 Décembre 1983 portant organisation du Ministère de la Jeunesse et des Sports mentionnait pour la première fois que le service Sport de masse qui englobe le SPT a pour charge notamment «de concevoir, planifier et mettre en ouvre en collaboration avec le mouvement sportif et les collectivités publiques des programmes d'activités physiques et sportives variées, ouvertes à toute la population, réalisée sous forme de récréations actives et de loisir ».

\section{STRUCTURES ET ORGANISATIONS DU SPT EN TUNISIE}

La politique SPT doit être intégrée dans la politique générale sportive et bénéficier d'autant que le Sport d'Elite d'un budget conséquent et d'une programmation dans le cadre du budget d'équipement et d'une formation de cadres adéquate.

Sa gestion et sa mise en œuvre ne peuvent être assumées par le MJS qui est appelé à encourager la création d'une organisation nationale et favoriser une collaboration et une coopération permanente et effective entre les services du sport de masse du Département et les organismes bénévoles car malgré son développement spectaculaire le SPT s'appuie encore sur le dynamisme farouche du bénévolat.

Cette structure nationale aura pour charge de mettre en œuvre la politique et la stratégie proposées et d'établir une coopération avec les fédérations affinitaires telles que l'ANSCT, les fédérations nationales dirigeantes ainsi que tous les autres secteurs qui constituent les différentes intervenants virtuels tels que l'habitat, la santé, les institutions socio-éducatives, les compagnes d'assurance, les caisses de sécurité et surtout les collectivités publiques locales et les conseils de gouvernorat (comités communaux et comités régionaux des sports).

La deuxième solution consiste à confier ces charges au Comité Olympique Tunisien qui réaliserait l'autre version de ses attributions ; une mission sportive qui complèterait d'une manière harmonieuse celle de la haute performance, de l'olympisme.

Organisme National ou Comité Olympique, l'essentiel réside dans l'organisation rationnelle des cellules de travail et leur adéquation aux nouvelles données du SPT.

D’une manière générale, les unités suivantes peuvent être créées :

- Unité de coordination entre les différents intervenants.

- Unité d'enseignement SPT (notamment au niveau des scolaires).

- Unité des compétitions populaires et des épreuves de forme physique.

- Unité de relations publiques.

- Une agence de publicité.

- Un bureau de consultation technique et organisationnel.

- Un conseil pédagogique et scientifique.

\section{SOCIALISATION ET SOCIALISATION SPORTIVE}

\section{LA SOCIALISATION}

On peut définir la socialisation comme l'intériorisation des normes et des références qui permettent de devenir membre d'un groupe social, de se percevoir comme tel, et d'agir de façon appropriée dans ce groupe.

17 - Slim veut dire en arabe sain de corps et d'esprit.

${ }^{18}$ - Conférence Nationale sur le Développement du Sport (31 Août 1981) : document rédige en arabe édité fin 1981 par le Ministère de la Jeunesse et des Sports de Tunisie ; p. 38 et 39.

19 - Education Physique et Sportive. 
Il faut souligner certains termes de cette définition :

- Intériorisation : ce n'est que très rarement un apprentissage conscient (quand on nous dit «ne parle pas la bouche pleine »). En général au contraire on apprend les normes parce que les autres autour de nous les appliquent. Ainsi on offre des poupées aux petites filles et des panoplies de cow-boy aux garçons. Une exception notable est la socialisation anticipatrice : c'est ce qui se produit quand nous nous préparons à entrer dans un groupe et essayons de nous conformer à l'image que nous en avons. Ainsi nous nous préparons pour la rentrée des classes, ou pour un nouvel emploi...

- Normes et références : il ne s'agit pas seulement de règles de conduite mais aussi tout ce qu'on peut mobiliser pour communiquer avec les autres, et tout ce que l'on peut tenir pour acquis, naturel : le langage SMS, les façons de s'habiller... ces normes et références forment la culture d'un groupe.

- Groupe social : il existe des groupes sociaux de taille différente, ils sont imbriqués les uns dans les autres ou se recoupent en partie. La société dans son ensemble possède ses normes, mais aussi les supporters d'un même club de foot, des voisins dans une résidence, un groupe d'amis, voire chaque famille peut avoir des normes propres. Par ailleurs ces groupes ne sont pas forcément «légitimes» : devenir un « déviant» aux yeux de la société nécessite aussi d'apprendre les normes des groupes de déviants !

On distingue souvent la socialisation primaire qui se fait dans la famille jusqu'à l'adolescence et qui forme la base de la personnalité, de la socialisation secondaire, qui se poursuit tout au long de la vie dans les différents milieux sociaux que nous sommes amenés à traverser : école, études, milieux professionnels, cercles de sociabilité, etc.

Des trois instances qui assurent traditionnellement la socialisation dans les sociétés modernes: la famille, l'école et les pairs, deux paraissent en crise à l'époque actuelle : les familles décomposées, déstructurées par le chômage, sont-elles encore capables de socialiser les enfants ? Les difficultés qui traversent l'institution scolaire ne sont-elles pas le signe que le message ou sa forme ne sont plus adaptés à leur public, et par suite échouent dans leur mission ? Ces questions très dans l'air du temps, mettent en relief un biais majeur : elles sont normatives au sens où elles ne considèrent comme socialisation que la "bonne » socialisation, qui est toute proche de la «bonne éducation », définie par certains groupes sociaux ou par des experts. Or même une famille décomposée socialise, même si le modèle est différent. La preuve en est, que l'expérience du divorce des parents qui pouvait être traumatisante il y a 30 ans est aujourd'hui banalisée (non sur le plan personnel mais dans la façon dont il affecte la relation aux autres) : par-delà les histoires individuelles qui peuvent être très dures, les familles ont appris à s'accommoder du divorce comme d'un événement qui a de grandes chances de survenir et non comme la preuve d'une tare personnelle. Ceci étant, il semble de plus en plus manifeste que dans la socialisation des jeunes l'école et la famille ont cédé du terrain au groupe de pairs (camarades de classe ou d'autres activités), qui concurrence de plus en plus l'autorité des enseignants et des parents.

Définition de Guy Rocher : «Ensemble de processus par lequel l'individu apprend des manières de faire, d'agir, de penser, de sentir c'est à dire la culture $»^{20}$.

La socialisation n'uniformise pas totalement. Tout au long de sa vie, l'homme évolue, sa personnalité sociale aussi évolue : l'homme est unique et uniforme. Le résultat est complexe.

\section{LES FONCTIONS DE LA SOCIALISATION}

Il y a deux conséquences :

Le conformisme : ce qu'on attend de l'individu, ce qu'il doit faire.

La déviance : écarts aux règles. Un déviant n'est pas forcément anticonformiste.

Il est important de distinguer plusieurs stades : La socialisation primaire : premier processus de socialisation d'un nouveau-né par l'intermédiaire de la famille, de l'école, des pairs, des média. Cette socialisation est importante car elle va apprendre à l'enfant à vivre en société. La socialisation secondaire:elle se superpose à la première forme de socialisation. Elle va se faire par l'entreprise, les pairs (les amis)... Dans cette dernière, il arrive des événements très importants comme le mariage, naissance du premier enfant, mariage de ce dernier,...

\section{FORMES DE SOCIALISATION}

Comment l'enfant acquiert-il les éléments de la culture ?

$\checkmark$ Par l'apprentissage :

Répétition : l'enfant répète les mêmes choses que les parents ainsi cela devient un réflexe, un conditionnement L'imitation : l'enfant imite ses parents.

Renforcement/coercition : l'enfant reçoit des récompenses/punitions et son apprentissage se structure en conséquence.

20 - GUY ROCHER, Introduction à la sociologie générale, Montréal (HMH) ,1968. 
$\checkmark$ Par l'intériorisation d'autrui :

Elle complète l'apprentissage. On insiste sur l'importance de l'interaction (rapport avec les autres). C'est par ce moyen qu'on acquiert un rôle.

Jean Piaget considère que jusqu'à 7 ans, l'interaction se fait surtout avec les parents (domaine de l'apprentissage). A partir de 7 ans, l'interaction se fait avec les groupes de pairs avec l'apprentissage de nouvelles normes, d'autres valeurs (vers une plus grande autonomie).

\section{LES AGENTS DE LA SOCIALISATION}

La socialisation se fait tout au long de la vie ,la famille,l'église,l'école,les syndicats,les entreprises, les associations,les parties politiques et les média, Ces agents de socialisation interviennent aux différents âges de la vie. On pourrait associer ces agents aux différentes de socialisation :Socialisation primaire : groupe des pairs, famille, école , Socialisation secondaire : entreprises, parti politique, associations...

Ces agents de socialisation peuvent avoir une fonction explicite ou implicite de socialisation.

Fonction explicite : la fonction principale de l'agent est de socialiser et elle concerne tous les domaines de socialisation.

Fonction implicite : la fonction principale n'est pas de socialiser. (Ex : associations sportives).

L'école a deux fonctions : un rôle d'éducation, un rôle d'intégration. Cela correspond aux deux illustrations qui suivent

Un rôle d'éducation : elle doit prévenir la violence dans certaines banlieues aujourd'hui. (Civilité, socialisation, la question du respect)

Un rôle d'intégration : Pour Durkheim, dès la fin du XIXème siècle, l'école participe au processus d'intégration culturelle. Elle oblige à une coupure entre les parlers breton, corse (de la maison) et le français, la langue de la France que l'on parlera à l'école et en public. De même, le principe de laïcité est affirmé au sein de l'école. On opère également une coupure entre la pratique religieuse de la famille et l'absence de référence religieuse dans le public. Tout cela participe à détacher l'individu de ses particularismes pour en faire un citoyen français.

La socialisation différentielle par rapport au milieu social donne naissance au style de vie.

Qu'entend-on par style de vie?

Style de vie : ensemble des pratiques de la vie quotidienne dont la cohérence provient du système de valeurs et de normes intériorisés par l'individu.

Ainsi le milieu d'appartenance influence les pratiques quotidiennes : c'est le style de vie.

\section{THEORIES}

Les théories de la socialisation sont définies par la tension entre l'intériorisation normative et culturelle et la distanciation critique. Elles s'efforcent d'expliquer comment les individus sont à la fois les membres d'une société et des acteurs autonomes. Il est possible de distinguer deux grandes familles théoriques selon qu'elles insistent sur l'une ou l'autre de ces dimensions. Par ailleurs, ces théories de la socialisation renvoient à deux grandes conceptions de l'intégration : les théories de l'intégration sociale et les théories de l'intégration systémique.

\section{A/L'intégration sociale}

Le modèle de l'intégration sociale suspend l'établissement d'un ordre et d'une régularité du système aux accords culturels et normatifs. Dans la mesure où la socialisation harmonise les conduites, les attentes, les capa cités d'adaptation des acteurs, elle est au fondement de l'ordre social. Le lien entre l'acteur et le système n'est pas donné, mais il est reconstruit dans et par les individus lors de leur socialisation. C'est en ce sens que la socialisation devient une sorte d'équivalent universel de la sociologie qui vise à établir des correspondances entre des positions sociales et des pratiques. La socialisation est à la fois ce qui explique les conduites et ce que les conduites doivent expliquer.

Pour des auteurs comme Parsons ou Habermas, et malgré la grande attention accordée aux processus d'intégration systémique, il est clair que la socialisation tient le rôle majeur dans le maintien de l'équilibre de la société. C'est la réversibilité de l'acteur et du système qui est au centre de cette représentation. La «personnalité» est une disposition forgée par la société, d'où la place tenue dans leurs œuvres, comme dans celle de Durkheim, par l'analyse des défaillances et des crises de motivation; par l'anomie, de quelque manière qu'on la nomme.

\section{B/ L'intégration systémique}

La seconde conception de l'intégration n'accorde pas un rôle central à la socialisation dans l'explication de l'intégration sociale. Certes, les auteurs sont loin de nier la réalité d'un processus de socialisation des 
individus, mais désormais celui-ci ne permet plus de structurer la société. La société est conçue, soit comme le résultat d'une agrégation, plus ou moins aléatoire, de différentes actions individuelles étayées par des motivations multiples, soit comme un enchevêtrement des domaines d'action sans principe central, dont l'accord n'est plus établi au niveau de la société toute entière, mais à partir de chaque système partiel en accord avec des réseaux communicationnels toujours conjoncturels. La société moderne opère un transfert du poids essentiel de l'intégration sociale vers des principes d'intégration systémique, vers des moyens de plus en plus impersonnels qui coordonnent les actions des individus en dehors de leurs arrangements culturels (N. LUHMANN, 1991 ; N. LUHMANN, R. De Georgi, 1993). On privilégie ainsi parfois l'étude des différents écarts entre les motivations des individus et les résultats des actions. Les situations objectives et «systémiques» s'autonomisent par rapport aux dimensions subjectives de l'action; la régulation des rapports sociaux ne peut plus être envisagée seulement au niveau local, elle exige la prise en compte d'un ensemble plus vaste et plus impersonnel, celui des mécanismes d'intégration systémique. Effet émergeant ou mécanisme systémique autonome, l'intégration du système ne peut pas être confondue avec les accords culturels et normatifs.

La socialisation est-elle un processus d'inculcation, d'apprentissage, d'intériorisation des fonctions sociales, d'incorporation d'un habitus ou de «construction sociale de la réalité »?... Voilà qui résume l'évolution des théories de la socialisation. Quatre moments de l'histoire des sciences sociales sur ce thème sont donc aisément identifiables : l'analyse psychologique de Piaget et la remise en cause de Durkheim, l'approche culturaliste (Benedict, Kardiner) et fonctionnaliste (la «suprême théorie » de Parsons), l'approche de Bourdieu et, enfin, les dernières analyses débutant dans les années 1980, et qui insistent sur l'incertitude de la réalité, le poids de l'acteur et de ses interactions avec autrui dans un contexte culturel donné.

\section{SOCIALISATION PAR LE SPORT}

« Nous définissons provisoirement la sociabilité comme les formes d'expressions et la régularité des manifestations et des relations qu'un individu entretient avec autrui dans un domaine constitué objectivable. ${ }^{21}$

«Le mot sport doit être immédiatement précisé par la notion d'équipe sportive qui permet de concevoir un espace de socialisation précis $»^{22}$

« Le sport peut être, pour les jeunes en difficulté, un lieu de resocialisation par dépassement des différences, retournement des stigmates, réappropriation d'identités positives $»^{23}$.

Selon Fize, la socialisation est « Le processus par lequel les individus apprennent les modes d'agir et de penser leur environnement, les intériorisent en les intégrant à leur personnalité et deviennent membres de groupes où ils acquièrent un statut spécifique »; ainsi, « Le club sportif est un espace de sociabilité. Il renforce les liens avec le groupe social, crée des réseaux de solidarité locale et de sentiments d'appartenance ${ }^{24}$.

Le sport prend de plus en plus de place dans notre société. L'ambiance de nos villes peut être complètement transformée les soirs de grands matchs, et les Jeux olympiques sont un événement digne de la mondialisation. Les médias l'ont bien compris : il y a des journaux consacrés aux sports, des chaines télévisées thématiques.

Le sport, n'est pas une religion. Déjà du temps des Romains et des Grecs, il suffisait d'un peu de pain et des jeux pour calmer le peuple (Panem et Circenses). Il ne faudrait pas que cette activité — si belle et si importante soit-elle - en vienne à assoupir les humains et à les détourner, par l'euphorie des sens, de la question du sens de l'existence...

Les valeurs humanistes restent-elles à l'honneur dans le sport moderne?

\footnotetext{
${ }^{21}$ - Acte du colloque des 14 et 15 octobre 1993 à Bordeaux. (1995) Sport, relations sociales et action collective. Chapitre sur " L'Espace des sociabilités féminines en sports collectifs ", par Christine Mennesson. Talence : éditions de la maison des sciences de l'homme d'aquitaine. P. 231-237.

${ }_{22}$ - Vulbeau, Alain (1993). L'équipe sportive, espace de socialisation? Dans "Du stade au quartier : Le rôle du sport dans l'intégration sociale des jeunes ", p.81

${ }^{23}$ - Dubar, Claude (1993). L'autre jeunesse : d'autres voies de socialisation? Dans "Du stade au quartier : Le rôle du sport dans l'intégration sociale des jeunes ", p.36.

${ }^{24}$ - Fize, Michel (1993). Socialisation- sociabilité : qu'en dire ?, dans "Du stade au quartier : Le rôle du sport dans l'intégration sociale des jeunes ", p.39.
} 
Tout d'abord, les valeurs humanistes sont présentes dans le sport moderne. Les adversaires se serrent la main, ce qui évoque le respect de l'autre. De plus, nous pouvons voir plusieurs protagonistes ce qui représente l'esprit d'équipe et le partage. En regardant leur visage, nous pouvons dire que le sport leur apprend l'épanouissement personnel et le développement de soi, ils se préparent à se dépasser. Enfin, nous ajouterons que c'est à leur âge que l'on apprend la différence entre le bien et le mal, les véritables valeurs dans la vie.

Puis, la problématique «les valeurs humanistes restent elles à l'honneur dans le sport moderne? » se pose en regardant plus attentivement la photographie. Les jeunes ne sourient pas, ils ne se serrent pas franchement la main et ils le font sans se regarder dans les yeux. Ce geste ne serait alors seulement le résultat de règles, d'habitudes ou de consignes des entraineurs?

Il existe de nombreuses définitions des valeurs humanistes. Il s'agit d'un mouvement d'esprit représenté par les humanistes de la Renaissance. C'est une philosophie et une vision du monde raisonnée et humaine. Son idée de base est tout d'abord orientée vers l'Homme, c'est-à-dire, poursuivre le bien-être et dignité. Deuxièmement, c'est la recherche de la vérité par l'intermédiaire de moyens humains, en particulier les sciences, en solidarité avec l'humanité. Troisièmement, c'est de la poursuite du sens de la vie, autrement dit le soin des gens et le respect des valeurs humaines.

En parlant des prémices du sport, tout le monde pense immédiatement aux Jeux Olympiques. On sait qu'ils se sont originellement tenus dans la Grèce antique du 8ème siècle avant notre ère. Au début, les JO avaient lieu dans le but d'éviter la guerre car les peuples anciens se promettaient de ne pas se battre au cours des $\mathrm{JO}$, donc, ils sont un symbole d'amitié et de paix du respect des règles, la tolérance des autres joueurs, la solidarité, l'acceptation de l'échec et ré-médiation, le dépassement de soi, et la volonté d'arriver à ses fins etc. Pendant ces compétitions-là, l'esprit sportif était bien respecté. C'est la présence des valeurs humanistes dans le sport! Mais aujourd'hui, est-ce que les valeurs humanistes restent-elles à l'honneur dans le sport moderne?

\section{LES FORMES DE SOCIABILITE DEVELOPPEES AU SEIN DE SPT}

\section{A/ Vie en groupe dans le SPT}

La sociabilité développée au sein de clubs de SPT serait commandée à l'origine par une finalité provoquée par les exigences de la vie. La prospérité ici exige une conjugaison des efforts. Il faut toujours mettre en commun les moyens matériels, humains et financiers pour prétendre monter un projet. L'activité sportive de plaisance, la pratique de tontine, l'assistance apportée à un membre dans les moments de joie ou de peine, l'assistance apportée aux nécessiteux de tous ordres nécessitent un investissement.

On peut dire que le SPT organisé dans l'espace improvisé organisé a permis la mise en commun, à moindre coût, d'un lieu d'exercice indispensable pour l'activité.

Le matériel nécessaire pour cette activité n'aurait jamais pu être acquis individuellement par les membres. On peut retenir l'idée que le groupe de SPT ainsi composé de (chômeurs, commerçants, professeurs, ingénieurs, médecins, etc.), et pas seulement d'abonnés est une institution moderne en ce sens qu'ils participent dans un autre cadre, selon Maurice Agulhon, à une certaine « collectivisation de la vie ».

Les activités des groupes improvisés organisés de SPT ne sont pas restreintes à la proposition d'activités physiques ou culturelles. Si nous définissons la culture comme ce qui rassemble les hommes pendant quelques nuits et quelques jours, ce qui remodèle notre perception des lieux, ce qui nous soustrait à la dure loi de la réalité et à ses contraintes, ce qui abolit les différences d'âge, de sexe, de condition sociale, ce qui bouleverse nos sens et les emplit d'une ferveur oubliée. En ce sens, est ce que le SPT n'est pas une culture dans la mesure où, les membres de SPT développent un style de vie, une manière de faire du SPT avec des principes qui sont mis en application au sein du groupe ? On peut dire que le SPT est une microsociété.

\section{B/ SPT microsociété et d'échange généralisé.}

L'intégration des pratiquants de SPT dans la société par la participation active au monde du travail et la persistance des liens de solidarité entretenus entre les individus en dehors de la sphère sportive, crée des réseaux d'échanges, d'informations, de biens et de services, en même temps que des obligations et une interdépendance entre les bénéficiaires.

La connaissance de ces réseaux et de leur fonctionnement permet de comprendre le système de relations existant dans le SPT, et qui donnent à ce fameux esprit SPT, sa signification sociale. Mais, il existe d'autres types de biens d'échanges dont la possession et la circulation sont un facteur d'intégration à la société globale.

Par exemple le mot d'ordre fraternel d'un tel groupe «sourire aux lèvres ha.ha.ha», ponctue alors la rencontre. Cette manifestation autorisée par une reconnaissance mutuelle est de l'ordre du contact verbal. Une fois reconnus, les signes et gestes représentatifs d'un habitus commun, le mécanisme du réseau de solidarité 
peut être enclenché, car la communauté du SPT s'organise en une microsociété dont les membres sont présents dans tous les secteurs de la vie civile et professionnelle.

Le réservoir de biens et de services disponibles est donc très varié, le système d'échange se mettant en place au gré des circonstances et des occasions. Chacun peut normalement en bénéficier, autant dans sa vie privée que dans sa vie publique.

Il lui suffit de faire appel à son réseau de connaissances pour remonter jusqu'aux personnes détentrices du savoir faire, du bien matériel ou de l'influence désirés.

L'échange est généralisé, le contre don, ce qui est rendu «n'est tenu à aucune condition de temps, de quantité ou de qualité $»^{25}$, ainsi que l'écrit Marshall SAHLINS au sujet du don à l'intérieur du réseau primaire composé de proches.

Donneur et récepteur ne sont pas nécessairement les mêmes lors de la contre prestation. Celui qui a donné recevra d'un autre et ainsi de suite. Celui qui a reçu un objet, un service ou une faveur d'un individu est en fait redevable envers toute la communauté du SPT. Il sera peut- être, lui aussi, un jour sollicité en tant que membre du réseau d'entraide. Et puisqu'il appartient à la famille SPT, on fera appel à son esprit de famille. Pour reprendre Pierre Bourdieu pour qui : « la famille est le lieu de la confiance (trusting) et du don (giving) par opposition au marché et au donnant, ou, pour parler comme Aristote, de la philia, mot qu'on traduit souvent par amitié et qui désigne en fait le refus de l'esprit de calcul, le lieu où l'on met en suspens l'intérêt au sens étroit du terme, c'est-à-dire la recherche de l'équivalence dans les échanges ${ }^{26}$.

La microsociété du SPT transforme les services de la société marchande en objets d'un système de don et de contre don à l'intérieur duquel, comme ailleurs, l'intérêt ne réside pas tant dans la gratuité et la liberté du geste que dans l'entretien du lien social.

Le seul fait d'être membre du club SPT peut permettre aux pratiquants d'obtenir des faveurs de la part de certains détenteurs d'un pouvoir. Ils n'ont pas eu à être recommandés par des personnes appartenant au réseau ; ils n'ont pas eu besoin de passer par des intermédiaires ; ils sont entrés d'emblée dans un système.

La sociabilité du SPT sans cesse activée par ce système d'échange généralisé double d'un temps de pratique collective, sportive et d'ambiance de gaieté, se pérennise également grâce à une mémoire partagée.

\section{SOCIABILITE SPORTIVE}

Dans le contexte français, on définit les sociabilités comme «des pratiques des relations sociales qui font l'objet de normes de convenances sociales ». Il attribue deux sens à la sociabilité sportive : c'est, très généralement, le fait que les gens s'organisent pour pratiquer le sport (...). Et c'est aussi, plus généralement, le fait que à l'intérieur du club, les adhérents nouent, entre eux, des relations et pratiquent des rites de confraternité, de solidarité, voire d'amitié.

Le SPT obéit à la logique de la participation de tous sans condition de performance, sans barrière culturelle et ethnique, au sein de ses activités, les adhérents développent des formes d'entraide basées sur la tontine, l'assistance à un membre, les actions philanthropiques ; ce qui laisse penser que le SPT doit être appréhendé comme une pratique sociale et culturelle, socialement et inconsciemment façonnée par ceux qui s'y adonnent, et largement partagée pour que les individus engagent, de manière incorporée, les propriétés fondamentales de leur groupe. Si les individus acceptent d'engager leur corps dans une activité corporelle comme le SPT, c'est peut-être parce qu'il contribue à la construction de leur identité sociale. De ce point de vue, le pouvoir identitaire du SPT est profondément lié à l'inscription corporelle des pratiquants. Ce qui peut faire de l'espace SPT, de puissants symboles sociaux. Ces préalables ont pour objectif de montrer, que l'analyse des « processus de socialisation » est indispensable pour appréhender la dynamique sociale de l'espace du SPT, et d'un autre côté, la production de sens que chaque pratiquant engage dans son rapport avec le corps ne peut s'analyser qu'en relation avec la dynamique du groupe et de l'espace.

\section{A/ SPT ; espace de diversification et d'individualisation des pratiques}

\footnotetext{
25 - Marshall, Sahlins. Âge de pierre, âge d'abondance. L'économie des sociétés primitives, Edition Gallimard, Paris, 1976

${ }^{26-}$ - Bourdieu : Raisons pratiques. Sur la théorie de l'action, Editions du Seuil, Paris, 1994 P.136-137. 
Une première caractéristique des activités de SPT est de se développer en dehors des cadres institutionnels et parfois en opposition aux modes d'organisation en place. C'est le cas dans certaines activités physiques qui connaissent un essor important. Ce sont par exemple le cas des pratiques pédestres (marche, jogging, course) et du cyclisme qui s'affirme toujours comme une activité de premier plan dans la plupart des clubs SPT.

En dépit de l'essor des activités individuelles, aisément réalisables en dehors de tout cadre associatif, celui-ci se développe en se diversifiant. De l'association éphémère de quelques-uns autour de la réalisation ponctuelle d'une pratique jusqu'à l'organisation associative sophistiquée impliquant des moyens matériels et humains importants, se répartit toute une série de formes associatives intermédiaires, selon les modalités spécifiques des pratiques. L'association s'affirme alors comme un moyen d'élargissement des choix couplé aux satisfactions qu'elle apporte au plan de la sociabilité, tout en cherchant à minimiser les contraintes d'organisation qui sont sources de limitation du temps consacré à l'activité.

Le deuxième axe de la dynamique d'évolution est celui des acteurs sociaux qui s'impliquent dans les activités de SPT ; ils cherchent à agir en réalisant eux-mêmes l'activité ou les activités pour lesquelles ils affichent intérêt et passion. Les acteurs de cette forme sociale en constitution refusent de la vivre par procuration, ils veulent pouvoir pratiquer intensément le sport, sous différentes formes parfois, y compris celle de la compétition, tout en conservant pour la plupart une distance à l'égard de l'institution sportive. Ainsi, selon les moments et selon les circonstances, ils sont amenés à tester des pratiques nouvelles pour eux ou à renouveler la façon de les exercer, élargissant ainsi l'espace de leurs possibilités d'action.

\section{B/ SPT ; espace où se développent des logiques participatives}

Tout comme les activités physiques de compétition, les activités de SPT impliquent des choix, effectués pendant le temps disponible, mais à la différence des pratiques de compétitions, les activités de SPT n'impliquent ni la performance, ni des règles explicites. Dans ce type d'action, l'espace de jeu est extrêmement ouvert tant dans l'espace formel ou qu'improvisé.

Entre le choix de pratiquer ou de ne pas pratiquer une activité de SPT, la liberté semble totale. Pourtant, pour chacun, l'espace de choix apparait bien avec des limitations. Le sens du choix de ne pas pratiquer une activité de SPT, tout d'abord, s'est considérablement modifiée par l'affirmation croissante des pratiques des activités du SPT comme valeur (bien désirable), non seulement au sein des populations jeunes, mais aussi au sein des populations adultes.

En effet, la croissance du taux de pratique chez les adultes est telle que la possibilité concrète de choisir l'absence de pratique (ou la non pratique) tend à se réduire, compte tenu des multiples formes de sollicitations allant dans le sens de l'exercice d'une activité physique.

La détermination à l'exercice d'une activité se précise donc comme effet de l'affirmation croissante des pratiques des activités de SPT en tant que valeur ; alors que l'espace d'indétermination dans le choix des modalités de pratiques s'accentue au sens des activités du SPT. Pourtant, les limitations dans les choix restent très présentes chez les acteurs individuels. Elles sont perçues comme contraintes matérielles : lieux de pratiques, temps disponibles, coût de l'activité ; autant de limitations premières à l'exercice de certaines modalités pratiques, du savoir faire, nécessaire à l'entrée dans ces activités, en particulier pour les populations adultes.

Dans une dynamique d'expansion de la pratique des activités de SPT, tant par les types d'acteurs que par la gamme des activités, les formes organisationnelles générées par cet élargissement se multiplient. Elles vont de individuelle, familiale ou avec des amis, à l'organisation de service la plus sophistiquées où les pratiques physiques deviennent un service marchand, mettant en relation une clientèle, des techniciens d'activités physiques et des organisateurs.

De cette multiplicité de choix individualisés, il est cependant possible de dégager des tendances, nouvelles normes physiques, partiellement contrées par des « contre tendances ambiguës orientées vers l'avenir et vers le passé (néo-naturalisme, néo archaïsme, néo-tribalisme)» ${ }^{27}$.

27 - Morin Emile, sociologie, édition, Fayard, Paris, 1984, p.436. 


\section{C/ Le SPT ; un espace où se développent des logiques du spectacle}

Par leur visibilité croissante, dans les divers lieux et moments de la vie quotidienne, ces activités de SPT tendent désormais à s'imposer comme un des éléments constamment renouvelés du spectacle de tous les jours.

Intégrée aux « rencontres sociales ${ }^{28}$ de la vie quotidienne d'une grande partie de la population, cette forme de pratique constitue, pour les individus, un ensemble de spectacles impromptus, répétitifs qui sont autant des sollicitations et d'occasions de se familiariser avec des modalités en plein renouvellement. La mise en place des pratiques de SPT au quotidien dans les rues n'est pas une chose véritablement nouvelle ; elle était déjà à l'œuvre dans les années d'après l'indépendance, mais avec une fréquence moindre, surtout sous la forme sportive, et avec une mise en place réglementée, dans les lieux bien circonscrits. Avec l'affirmation sociale du modèle sportif, et sa diffusion, le spectacle sportif dans les activités de SPT est devenu une forme essentielle de sensibilisation d'une grande masse d'acteurs sociaux à ces pratiques.

Offrir un spectacle attrayant au public, directement ou par la médiation des moyens de diffusion, écrits, parlés et visuels, implique pour les organisateurs, dirigeants des clubs, une spécialisation dans cette fonction. Mais la qualité du spectacle dépend directement de celle des acteurs et leur capacité d'action impliquant des savoir faire corporels sortant de l'ordinaire afin qu'ils réalisent l'activité et en déterminent la qualité. Ces ressources humaines sont donc à la fois, comme dans le cadre de pratique de compétition dont ce type d'action est essentiellement issu, des objets de l'action qu'il faut préparer, façonner, polir, par l'acquisition de techniques et de capacités corporelles hors du commun. Ce sont en même temps des sujets ayant déjà effectué des choix, en particulier celui du SPT comme une valeur marchande et une qualité de leur savoir-faire.

Quel est donc ce public sans lequel il n'y a pas de spectacle ? Ce sont les mêmes qui, à des titres divers, étudiants, compétiteurs, pratiquants dans le cadre participatifs, s'adonnent par ailleurs à des activités, pour certaines identiques ou similaires, dans leur dénomination et leurs règles formelles. Ce sont aussi ceux qui, anciens pratiquants dans l'un des cadres précédemment identifiés, ou même sans avoir jamais eu une connaissance pratique des activités choisies, sont devenus des connaisseurs par le spectacle de l'action. Que ce soit directement ou par médias interposé, le public est donc constitué d'acteurs sociaux opérant des choix : celui d'être des spectateurs assidus ou occasionnels ; celui de privilégier la dimension de l'émotion ou de la raison; celui d'apprécier la violence contenue ou la violence ouverte; celui de préférer les affrontements avec soi, avec l'autre ou avec l'environnement.

\section{D/ Le SPT ; un espace de développement de la personnalité du pratiquant}

Nous empruntons à P.Dolivet, la notion de «risque dynamique » dans la pratique physique et sportive. Le risque dynamique est une notion essentielle de l'individu en quête de liberté. Il est lié au sens que le sujet donne à l'acte risqué, à la justification partagée ou non, du risque pris. Une forme de violence qu'un pratiquant de SPT pourrait porter sur lui serait de ne pas prendre de risque d'aller pratiquer du sport, de rester sur le risque passif, c'est-à-dire de refuser de vivre quelques actions que ce soit sous prétexte qu'on n'en voit pas le sens, que l'on préfère la sécurité en restant non engagé dans une pratique sportive de SPT.

Mais, comme la participation de ce dernier peut permettre le rituel de conciliation sociale qui pourra favoriser la résolution d'une tension durable entre le sujet et le groupe à travers la pratique sportive, il ne peut s'en empêcher. Ainsi, le risque dynamique oblige le pratiquant à choisir, à se prendre en charge, à échapper au confort sécuritaire qui élimine les espaces de liberté sous prétexte d'éliminer toutes les possibilités d'accidents fâcheux. Par son action consciente, le pratiquant passe du probable au possible, il acte son projet, le concrétise avec prudence. Le risque dynamique serait de glisser du défensif à la prévention offensive, celle qui mettrait à disposition des espaces plutôt que de gérer et contrôler leur utilisation. Cela induirait la mise en œuvre de synergies, d'outils communs, même si parfois quelques dérives apparaissent. La restructuration de la personne, sa réinscription dans un univers social passe par un étayage du «moi » à travers l'émergence de la personne des autres. En ce sens, le pratiquant du SPT devra trouver et expérimenter sa solution dans le risque (faire du milieu son milieu-même à travers la communication à autrui et à un défi accepté à la société).

\section{E/ Le SPT ; un espace de voies d'intégration sociale}

Les activités physiques et sportives et le «sport pour tous » en particulier, sont perçus comme des moteurs et des voies d'intégration sociale à travers des objets tels que la solidarité, la responsabilité, l'autonomie, etc.

28 -E. GOFFMANN « La mise en scène de la vie quotidienne ». Tome 1 et 2, édition, minuit, Paris, 1973. 
Le pratiquant du «sport pour tous » qui a perdu son emploi et est en chômage, ou bien le retraité qui ne bénéficie que d'une infime partie de sa pension retraite, ou même celui qui n'arrive même pas à toucher sa pension de retraite...

C'est aussi, dans le pire des cas, un être (homme ou femme) qui a été lésé et qui est victime d'injustice sociale. Donc l'acte délictueux ici est un acte de révolte contre l'injustice subie. C'est, en tout cas, ce que le pratiquant exprime lorsqu'il tente de légitimer son comportement. Nous constatons bien qu'avec des injustices, l'individu se trouve de plus en plus placé dans un environnement stressant. Le bon fonctionnement du système nerveux est compromis par l'absence de calme, le manque de sommeil et l'incohérence des rythmes de vie. Ceci peut provoquer automatiquement un manque de contrôle de soi et un passage à l'acte immédiat, souvent sans autre finalité que la décharge d'énergie, la recherche d'indépendance à travers des comportements anomiques d'opposition et de défi. Ces personnes aux comportements asociaux ont besoin d'un groupe du genre SPT pour reconstruire leur identité à travers la vie en commun et le mélange de cultures qu'il encourage. Le SPT peut ainsi être considéré comme un système de normes, de valeurs, que les individus vont utiliser en s'engageant dans les activités de leur choix.

\section{Conclusion de la partie théorique}

Si nous voulons résumer les caractéristiques des pratiques sportives de SPT pour les comparer à celles du sport traditionnel (sport de compétition), il convient de synthétiser les différentes dimensions de ces formes de pratiques sportives.

Il est vrai que les activités SPT réalisées pendant le temps libre de la vie quotidienne reflètent un nouveau souci du corps ; cependant, il ne faut pas oublier que la pratique des activités physiques libres se fait entre les individus, qui ont besoin d'être avec les autres, et de vivre en symbiose. Or l'harmonie du groupe ne peut exister que si le groupe fixe des droits et devoirs de chaque membre ; ce qui implique que le processus devrait intégrer des règles et normes qui indiquent progressivement la ligne de conduite de chaque pratiquant. C'est ainsi que le SPT est devenu une activité organisée, mise en place dans un espace formel et informel par des individus pratiquants, où ils développent des nouvelles formes de sociabilité sportives différentes de celles qu'on retrouve habituellement au sein des activités physiques et sportives traditionnelles.

En reprenant chacune des dimensions du concept de forme de pratique sportive, on constate que l'activité sportive de compétition s'oppose, point par point, aux pratiques sportives de SPT. Quand d'un côté la sociabilité est choisie, de l'autre, elle est forcée. Quand d'un côté on se déplace sur des lieux sportifs non standardisés, de l'autre, on se déplace sur des lieux très standardisés. Quand les pratiquants de SPT présentent un rapport au temps discontinu et circulaire (téléologie de la détente), la pratique compétitive renvoie à une conception linéaire du temps (le progrès). Quand d'un côté on pose le principe de l'égalité des chances de participer, de l'autre, on insiste sur les inégalités (fondée sur les compétences sportives nécessaires pour faire gagner le club). Quand d'un côté, on insiste sur la dimension duelle de l'activité, de l'autre, on choisit de privilégier la dimension collectivz

L'intérêt de notre étude consistait à repérer ces différentes formes de sociabilité liées aux pratiques sportives dans les clubs de «SPT » et de décrire comment elles se constituent, autour de quel intérêt, comment se font et défont ces formes de sociabilité. Et de voir si ce ne sont pas ces formes de sociabilité qui sont à l'origine de l'engouement des pratiquants vis-à-vis des activités SPT ces dernières années alors que dans les années d'indépendance, la valeur éducative de cette activité n'était pas partagée par tous.

\section{LA DESCRIPTION DU CADRE DE RECHERCHE}

\section{A. Définition de la population et de l'échantillon :}

Pour étudier les propos des pratiquants et animateurs concernant la socialisation des activités physiques libres, il nous fallait nous centrer sur la population des pratiquants et animateurs au niveau de nos institutions.

Un ensemble de pratiquants et animateurs ont donc été sollicités sur Grand Tunis. Au final, l'échantillon se compose de 808 personnes au niveau du «Grand Tunis », dont 352 pratiquants, 178 non pratiquants et de 121 animateurs et 157 responsables, on a essayé d'obtenir une participation la plus large possible, avoir un échantillon varié qui prend en compte pratiquants et animateurs exerçant à des salles de culture physique, a des parcours de santé. Et collecter des réponses de la part des personnes d'âges, d'ancienneté, de profession et de divers plans.

\section{B. Méthodes et moyens de collecte des données : \\ 1. Présentation du questionnaire :}

Notre questionnaire est composé de 191 questions variées adressées aux (pratiquants et animateurs,non pratiquants,responsables des structures sportive )dont quatorze portants sur des informations générales 
personnelles et des questions sollicitant les propos des pratiquants et animateurs sur l'impact de la pratique physique libre sur les différents plans individuel, collectif et pratique ainsi que sur les différents niveaux de la socialisation sportive. On va seulement interpréter les 31 questions les plus intéressantes.

\section{Lieu :}

Le questionnaire a été proposé à des pratiquants et animateurs occupant nos salles de sport et de culture physique et les parcours de santé, au niveau de 4 départements du « Grand Tunis » : La Manouba, Ben Arous, Ariana, et le département de Tunis.

\section{Passation et difficultés :}

Ce travail a été réalisé par l'intermédiaire d'une enquête sous forme de questionnaires, distribués à des pratiquants et animateurs des activités physiques libres qu'on a visité au niveau des établissements appropriées où ils exercent leur fonction, cette enquête c'est déroulé pendant le $2^{\text {ème }}$ semestre de l'année scolaire 2010-2011 sur plusieurs semaines, le pratiquant ou l'animateur disposera de quelques minutes pour répondre au questions en cochant la case de hypothèse qui convient pour chaque question.

On a seulement été retardé par les mesures nécessaires pour le contact des pratiquants, animateurs et les responsables afin d'accéder aux salles de sports et parcours de santé.

\section{Les buts du questionnaire :}

On a présenté ce questionnaire aux pratiquants et animateurs de SPT, dans le but d'avoir une réponse aux hypothèses, en référence à notre problématique, établies au préalable tout au début de l'étude.

La collaboration de nos pratiquants et animateurs questionnés sur « Grand Tunis » reflètera la situation actuelle de la pratique des activités physiques libres.

\section{5. choix des outils statistique d'investigation :}

\subsection{L'outil statistique :}

On utilise l'épreuve de khi carré ${ }^{29}$ pour comparer des effectifs ou des fréquences. On consigne ces effectifs avec leurs pourcentage dans des tableaux à double entrée appelés tableaux de fréquence ou contingence et croisant les variables indépendantes avec les variables dépendantes. Seulement, la façon de construire ces tableaux et les calculs à faire varient suivant que les échantillons observés sont indépendants ou appariés.

Nous avons deux traitements statistiques à faire, le premier qualifié de global permet de décider si la différence entre les fréquences positives (réponse par oui) et les fréquences négatives (réponse par non) est significative (HS) ou non significative sans tenir compte de la variable.

\section{CARACTERISTIQUES DE L'ECHANTILLON}

\section{INTERPRETATIONS DES RESULTATS}

$\Rightarrow$ Notre échantillon se caractérise par :

> Selon l'âge : nous avons un échantillon majoritaire d'une tranche d'âge entre 20 et 40 ans.

$>$ Selon le sexe : on n'a pas une différence entre les hommes est femmes puisque le pourcentage des deux sexes est proche avec un léger décalage du sexe masculin et c'est peut être parce qu'on vit dans une société masculine.

> Selon la profession : la majorité de notre échantillon sont des étudiants puis les employés alors que la minorité sont les retraités, cette grande participation des étudiants et employés est peut être dû au temps libre disponible chez eux d'où ils trouvent dans la pratique sportive un moyen d'évasion.

> Selon l'état civil : nous avons un échantillon de majorité célibataire d'où ils occupent leurs temps libre dans une pratique sportive à fin d'entretenir leurs corps.

$>$ Selon le niveau scolaire : nous avons un échantillon dont la majorité a le niveau universitaire.

$>$ Selon les diplômes obtenus : la majorité de notre échantillon ont un diplôme de baccalauréat et/ ou de maîtrise.

> Selon leurs origine : heureusement que la majorité de notre échantillon sont originaires de la zone de Tunis car ceci a aidé le déroulement de notre recherche.

$>$ Selon le lieu de résidence : la majorité de notre échantillon réside dans des milieux communaux ce qui est peut être dû à la disponibilité des espaces de pratique dans ces milieux.

$>$ Selon la pratique d'une APS : la majorité de notre échantillon pratique une APS.

\footnotetext{
${ }^{29}$ Parlebas P, Cyffers B. Statistique appliquée aux activités physiques et sportives INSEP,France, 1992,p
} 
> Selon le lieu de la pratique de l'APS : la majorité de notre échantillon pratique l'activité physique dans un espace officiel.

> Selon les années de pratique: nous avons un échantillon dont la majorité a commencé la pratique récemment (-5ans).

> Selon leurs appartenance : plus que la moitié de notre échantillon sont affiliés à des institutions, et en second lieu on trouve qu'ils appartiennent à des associations.

> Selon le type de pratique proposée : on a un échantillon dont la majorité exerce une pratique collective.

$>$ Selon la participation dans l'élaboration des programmes SPT : la majorité de notre échantillon ne participe pas dans l'élaboration des programmes SPT qu'organise la fédération, la commune ou l'institution.

\section{ANALYSE ET INTERPRETATION DES RESULTATS DE LA $1^{\text {ère }}$ HYPOTHESE}

La sociabilité développée au sein de clubs de SPT serait commandée à l'origine par une finalité provoquée par les exigences de la vie ; on peut déduire que la sociabilité du SPT sans cesse activée par ce système d'échange généralisé double d'un temps de pratique collective, sportive et d'ambiance de gaieté, se pérennise également grâce à une mémoire partagée.

On conclus que la pratique des activités physiques libres a une influence positive sur la socialisation puisqu'elle favorise la communication inter-pratiquant et met en valeurs les relations humaines a travers l'instauration d'une bonne ambiance de pratique par le biais des compétitions organisés ainsi que l'acquisition de différentes valeurs morales comme la solidarité, la responsabilité l'autonomie et l'entre-aide ; et n'oublions pas le rôle que joue les programmes SPT dans l'acquisition des habiletés mentales et physiques chez l'individu.

\section{ANALYSE ET INTERPRETATION DES RESULTATS DE LA $2^{\text {ème }}$ HYPOTHESE}

L'intégration des pratiquants de SPT dans la société par la participation active au monde du travail et la persistance des liens de solidarité entretenus entre les individus en dehors de la sphère sportive, crée des réseaux d'échanges, d'informations, de biens et de services, en même temps que des obligations et une interdépendance entre les bénéficiaires. La connaissance de ces réseaux et de leur fonctionnement permet de comprendre le système de relations existant dans le SPT, et qui donnent à ce fameux esprit SPT, sa signification sociale.

On conclut donc que La pratique du SPT a un impact meilleur que la pratique du sport civil puisqu'elle favorise l'intégration dans le groupe car c'est un espace où se développent les logiques participatives ainsi qu'elle favorise la consolidation des relations sociales; n'oublions pas qu'elle aide dans la gestion et la réduction du stress, de l'anxiété, de la dépression et accroit la capacité de production de l'individu dans son travail.

\section{CONCLUSION GENERALE}

Comme on l'a déjà signalé auparavant, la sociabilité développée au sein de clubs de SPT serait commandée à l'origine par une finalité provoquée par les exigences de la vie ; on peut déduire que la sociabilité du SPT sans cesse activée par ce système d'échange généralisé double d'un temps de pratique collective, sportive et d'ambiance de gaieté, se pérennise également grâce à une mémoire partagée; d'où on peut dire que la pratique des activités physiques libres a une influence positive sur la socialisation puisqu'elle favorise la communication inter-pratiquant et met en valeurs les relations humaines a travers l'instauration d'une bonne ambiance de pratique par le biais des compétitions organisés ainsi que l'acquisition de différentes valeurs morales comme la solidarité, la responsabilité l'autonomie et l'entre-aide ; et n'oublions pas le rôle que joue les programmes SPT dans l'acquisition des habiletés mentales et physiques chez l'individu.

Aussi, l'intégration des pratiquants de SPT dans la société par la participation active au monde du travail et la persistance des liens de solidarité entretenus entre les individus en dehors de la sphère sportive, crée des réseaux d'échanges, d'informations, de biens et de services, en même temps que des obligations et une interdépendance entre les bénéficiaires.

La connaissance de ces réseaux et de leur fonctionnement permet de comprendre le système de relations existant dans le SPT, et qui donnent à ce fameux esprit SPT, sa signification sociale, par là on peut dire que la pratique du SPT a un impact meilleur que la pratique du sport civil puisqu'elle favorise l'intégration dans le groupe car c'est un espace où se développent les logiques participatives ainsi qu'elle favorise la consolidation des relations sociales; n'oublions pas qu'elle aide dans la gestion et la réduction du stress, de l'anxiété, de la dépression et accroit la capacité de production de l'individu dans son travail.

\section{RESUME}

On peut dire que c'est la logique du sérieux qui prime dans le sport de compétition et celle du loisir au sein du SPT. La majorité des pratiquants attendent un mieux être en général (physique, moral et social etc.), plus précisément, celui-ci est à la convergence du plaisir, de se mouvoir, de lutter contre les maladies cardio- 
vasculaires et d'être avec les autres etc. ; Ces causes multiples tiennent peut être à l'évolution des modes de vie dans la société Tunisienne qui est une société de plus en plus urbanisée et dans laquelle le temps de loisir est devenu une réalité pour une grande partie de sa population ; le corps est devenu l'objet de mode et sujet de préoccupation ; il faut l'entretenir, lui donner une beauté, l'affermir, le rendre tonique et sain.

Il est vrai que les activités SPT réalisées pendant le temps libre de la vie quotidienne reflètent un nouveau souci du corps ; cependant, il ne faut pas oublier que la pratique des activités physiques dans les différents clubs de SPT que nous avons eu à observer, se fait entre les individus, qui ont besoin d'être avec les autres, et de vivre en symbiose. Or l'harmonie du groupe ne peut exister que si le groupe fixe des droits et devoirs de chaque membre ; ce qui implique que le processus devrait intégrer des règles et normes qui indiquent progressivement la ligne de conduite de chaque pratiquant. C'est ainsi que le SPT est devenu une activité organisée, mise en place dans un espace formel et informel par des individus pratiquants, où ils développent des nouvelles formes de sociabilité sportives différentes de celles qu'on retrouve habituellement au sein des activités physiques et sportives traditionnelles.

. Le SPT privilégie les prouesses techniques individuelles, la virtuosité et l'esthétique du geste sans effort alors que le sport de compétition donne le primat à l'efficacité collective de la tactique, aux résultats acquis grâce à l'entraînement, à l'assiduité, à une certaine ascèse.

\section{RECOMMANDATIONS}

Il semble évident que dans le sport ce n'est pas la couleur de la peau qui compte mais celle du maillot. Le maillot habille une appartenance à une même « famille » et déshabille des clivages de la société. Le sport rassemble se que les sociétés oppose. Par le sport on apprend beaucoup, tout d'abord à vivre avec autrui, ensuite on apprend à respecter des obligations mais aussi des interdits imposés par des règles, des valeurs et des normes. On les retrouve notamment dans les règlements qui régissent le code du sport (fédéré et associatif), de même que si la règlementation n'est pas toujours écrite comme pour les sports de rues elle coule de soi. On peut donc conclure que le sport apporte un habitus aux jeunes dans leur socialisation. L'habitus est une manière d'intérioriser (ou d'incorporer) des normes et des valeurs propres à son environnement, à son groupe social de référence constitué par la famille, les amis (groupe de pairs), l'école, etc. L'habitus produit de la conduite et du rapport social.

En ce qui concerne les activités physiques libres ne constate pas de logiques de fonctionnement fondamentalement différentes entre les pratiquants de SPT. Ce qui veut dire que les configurations spatiales et socio-économiques ne sont pas premières dans la construction des formes des sociabilités des pratiquants de SPT. En fait, lorsqu'on, fait les croisements des variables liées aux sociabilités (tontines, type d'assistance à un membre,) avec les variables liées aux intérêts de la pratique etc.., ils ne mettent en évidence aucune relation particulière. Autrement dit, on repère les mêmes logiques sportives sur les deux villes.

Nous pouvons dire que notre recherche se trouve sur un territoire de plus en plus ambigu. Ambigu parce que, aux yeux du lecteur, elle suscite beaucoup de questions et d'interrogations. Si nous nous intéressons au corps vieillissant, qui se prépare à mourir plus ou moins rapidement, mais où la conscience de cette mort devient peu à peu incontournable, on peut dire que le corps vieillissant perd sa jeunesse, sa force, sa résistance. La plupart des modèles de la santé, nous présentent un concept du sport comme élément de la prévention. Nous pourrions dire schématiquement le slogan suivant : " pratiquons du sport pour reculer les limites de la décrépitude, du vieillissement, du déclin, des dégradations tant physique, physiologique, biologique, neurologique et psychique. Pratiquons du sport pour survivre, en bonne santé, le plus tard possible. ». Nous nous trouvons ici dans une logique de « quantité »: toujours plus d'années de vie, toujours plus d'entraînement (de préférence aérobic), garder, retenir la forme, avec toujours dans la tête, cette forme physique, esthétique, psychique présentée par les médias, une forme-modèle, toujours moins réaliste pour la personne vieillissante. Est-ce bien là ce que notre modèle de pratique sportive doit offrir ? Nous faisons l'hypothèse qu'il existe un autre modèle et d'autres explications à toute pratique sportive de l'âge avancé.

Nous considérons les différents groupes de pratique du SPT à la fois comme étant les clubs de sports ouverts à tous les pratiquants, à des pratiques les plus diverses, des groupes de pratiques au sein desquels se développent de nouvelles manières de vivre en société par opposition aux associations de sport d'élite qui ont été conçus explicitement pour les sportifs de haut niveaux.

\section{BIBLIOGRAPHIE}

[1] -Histoire du sport en tunisie : fr.wikipedia.org/wiki/Sport_En_Tunisie www.toutelatunisie.com/doc.php?docid=510

[2] -Définition de la socialisation : http://www.melchior.fr/2-1-Definition-de-la-socialis.3714.0.html

[3] R.Boudon (1997), dictionnaire de sociologie, 
[4] http://aees.free.fr/docs\%20liens/cours/12\%20semestre\%202/SHS\%20TATU\%20UE4A\%20Sport\%20et\%20socialisation.pdf

[5] Parlebas, Contribution à un lexique commenté en science de l'action motrice, Paris, INSEP, 1981.

[6] -Parlebas Pierre, Cyffers B. Statistique appliquée aux activités physiques et sportives INSEP, France, 1992.

[7] Le Grand Dictionnaire Larousse Encyclopédique.

[8] MATVEIEV, la base de l'entraînement, Vigot, Paris, 1980.

[9] LUSCHEN \& SAGE, Handbook of social science of sport, Stipes publishing company, 1981

[10] Document du Conseil de l'Europe, Charte Européenne du Sport pour Tous, p. 3 du document.

[11] Charte Européenne du Sport pour Tous, document CCC/EES (68) 10 rév. II Strasboug 1979.

[12] Cleaning House : Centre d'information sportive du Conseil de l’Europe, fiches d'informations 1974 , fiche $n^{\circ} 3$.

[13] Michel Paul BERNARD : professeur Bulletin FIEP, Mars 1981, pages 45 et suivantes.

[14] Simmel.G, sociologie et épistémologie, PUF, Paris, 1981.

[15] Définition intégrale, tiré du dictionnaire des APS, éditions Amphora 1998.

[16] GUY ROCHER, Introduction à la sociologie générale, Montréal (HMH) ,1968

[17] Abdelwahab CHAHED, Pour une politique Tunisienne de Sport pour Tous, Tunis-La Kasbah 1986.

[18] Document du Conseil de la Coopération Culturelle Sport pour Tous, 1970, cinq pays répondent : République Fédérale Allemande/ Pays-Bas/ Norvège/ Suède et Royaume Unie

[19] Charte Européenne du Sport pour Tous, document CDDS (79/88) résolution (76) 41 du Comité des Ministres Européens Strasbourg 1978 ,

[20] Conférence Nationale sur le Développement du Sport (31 Août 1981) : document rédige en arabe édité fin 1981 par le Ministère de la Jeunesse et des Sports de Tunisie .

[21] Fiche du Cleaning House N8303. 1107.0136. Avantages économiques de la pratique régulière d'une activité physique (Australie).

[22] -Document du VIe Plan de développement (1982-1986). Education.

[23] Joffre DUMAZEDIER, ancien Président du Comité de recherches sur le loisir de l'Association Internationale de Sociologie ; Le Monde du 6 Août 1984, Tribune Feu «Le temps libre ».

[24] Colloque International, Place des activités physiques et sportives dans le monde du travail, Bizerte, Novembre 1981, organisé par l'ANSCT Tunisie.

[25] -Pierre BOURDIEU, Le sens pratique, Paris, Éditions de Minuit, 1980.

[26] -Pierre Bourdieu, La Distinction, Minuit, 1979.

[27] -Bourdieu : Raisons pratiques. Sur la théorie de l'action, Editions du Seuil, Paris, 1994.

[28] -Lacan , citation : http://jaysesblogs.blogspot.com/2006/11/chapitre-la-socialisation.html

[29] Acte du colloque des 14 et 15 octobre 1993 à Bordeaux. (1995) Sport, relations sociales et action collective. Chapitre sur " L'Espace des sociabilités féminines en sports collectifs ", par Christine Mennesson. Talence : éditions de la maison des sciences de l'homme d'aquitaine.

[30] Vulbeau, Alain (1993). L'équipe sportive, espace de socialisation? Dans "Du stade au quartier : Le rôle du sport dans l'intégration sociale des jeunes "

[31] Dubar, Claude (1993). L'autre jeunesse : d'autres voies de socialisation ? Dans "Du stade au quartier : Le rôle du sport dans l'intégration sociale des jeunes ".

[32] Fize, Michel (1993). Socialisation- sociabilité : qu'en dire ?, dans "Du stade au quartier : Le rôle du sport dans l'intégration sociale des jeunes ".

[33] Marshall, Sahlins. Âge de pierre, âge d'abondance. L'économie des sociétés primitives, Edition Gallimard, Paris, 1976.

[34] Morin Emile, sociologie, édition, Fayard, Paris, 1984.

[35] -E. GOFFMANN « La mise en scène de la vie quotidienne ». Tome 1 et 2, édition, minuit, Paris, 1973. 